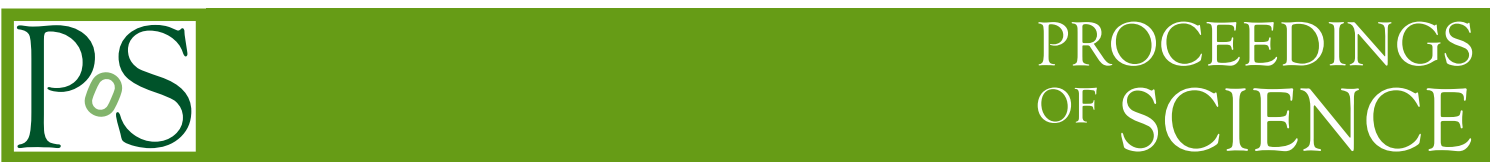

\title{
New detectors for new light sources
}

\section{Lodovico Ratti*}

Università degli Studi di Pavia and INFN

E-mail: lodovico.ratti@unipv.it

In the next few years, X-ray free electron laser (FEL) facilities are expected to proliferate all around the world. These $4^{\text {th }}$ generation light sources, based on linear accelerator technology, can provide coherent X-ray beams, femtosecond pulses and a brilliance ten orders of magnitude larger than storage ring based sources. New detectors will be needed to to take advantage of their features and let them fully deploy their probing power. This paper shall review the state of the art in the field of 2D X-ray imaging instrumentation, focusing on a selection of the large set of detector technologies and architectural solutions proposed for experiments at FELs. This will include some recent outcomes from the PixFEL project, an R\&D INFN-funded program aiming at the development of advanced X-ray imagers for applications at FEL sources.

INFN Workshop on Future Detectors

16-18 December, 2015

Aula Magna della Cavallerizza Reale, Torino, Italy

\footnotetext{
*Speaker.
} 


\section{Introduction}

The use of large accelerator-driven X-ray sources such as synchrotron light sources and X-ray free-electron lasers (FEL) continues to grow and expand to many scientific disciplines worldwide. These facilities are now driving the state of the art of X-ray science, therefore shaping the requirements for many types of X-ray detectors. X-ray FELs in particular can offer unprecedented capabilities in penetrating the microscopic structure of organic and inorganic systems, new materials and matter under extreme conditions and in recording and understanding the time evolution of fast biochemical phenomena at the nanoscale [1]. The emergence of these powerful, high luminosity sources is extending the diffraction method, already employed in X-ray crystallography, to the analysis of small, non-periodic specimens, such as single biomolecules or cells. Coherent X-ray diffraction imaging (CXDI) of non-periodic structures actually requires a remarkable increase in the source brightness (in excess of $10^{33}$ photons $/ \mathrm{s} / \mathrm{mm}^{2} / \mathrm{mrad}$ ) to compensate for the lack of the Bragg peak amplification mechanism typical of crystal diffraction [2]. This results in a huge amplitude range of the charge signal at the input of the system, which may exceed $80 \mathrm{~dB}$, and in an extremely large dose of ionizing radiation, in the order of few hundreds of MGy per year, deposited in the detector. Ultra-short X-ray pulses provide the needed resolution $(<1 \mathrm{ps})$ for time-resolved experiments, where a time dependent process is reconstructed using pump-probe techniques. In addition they can be exploited to extend the conventional radiation damage limit and record a scattering pattern from a single molecule before the sample eventually undergoes Coulomb explosion as a result of the large amount of energy released in it by the probing beam [3]. Actually, a single pulse is generally not enough to obtain a diffraction pattern with the required resolution to determine the structure of the specimen. Therefore, many identical copies of the same pattern have to be collected, among several millions of diffraction images, to provide statistically significant information [4]. In order to expedite the collection of the needed amount of data and improve the productivity, operation at very fast pulse rates, even exceeding $1 \mathrm{MHz}$, is foreseen for the next generation FELs. With these features, X-ray FELs promise to revolutionize several research fields, including structural biology and chemistry, materials science and nuclear and molecular physics, or to start entirely new ones. New technologies currently under development, such as those based on energy recovery LINACs [5], inverse Compton scattering techniques [6] and laser wakefield accelerators [7], may lead to the design of even cheaper and more compact systems, further boosting the construction of new X-ray (or gamma-ray) sources. In this scenario, continuous improvement on the existing instrumentation for the different experimental needs at FELs becomes of crucial importance to fully benefit of the outstanding properties of these powerful probing tools.

\section{Main features of X-ray FEL facilities}

As already mentioned in the introduction, FEL facilities provide high intensity beams of ultrafast X-rays, with an energy range from $100 \mathrm{eV}$ to more than $10 \mathrm{keV}$, corresponding to wavelengths between $10 \mathrm{~nm}$ and less than $0.1 \mathrm{~nm}$. Most of the measurements performed at FEL experiments are based on the scattering of coherent X-rays and the detection of the coherent diffraction pattern. More details about the experiments and measurement techniques deployed at FELs can be found in conceptual and technical design reports and publications easily found on the World Wide 


\begin{tabular}{|l|c|c|c|c|c|}
\hline Project & $\begin{array}{c}\text { Start of } \\
\text { operation }\end{array}$ & $\begin{array}{c}\text { Electron } \\
\text { beam energy } \\
{[\mathbf{G e V}]}\end{array}$ & $\begin{array}{c}\text { Photon Energy } \\
\text { /Wavelength } \\
{[\mathbf{k e V} / \AA]}\end{array}$ & $\begin{array}{c}\text { Burst } \\
\text { repetition rate } \\
{[\mathbf{H z}]}\end{array}$ & $\begin{array}{c}\text { Number of X-ray } \\
\text { pulses/train } \\
\text { @inter-train period }\end{array}$ \\
\hline FLASH@DESY & 2005 & 1.25 & $0.03-0.3 / 40-400$ & 5 & $800 @ 1 \mu \mathrm{s}$ \\
\hline LCLS@SLAC & 2009 & 14.5 & $0.3-10 / 1-400$ & 120 & 1 \\
\hline SACLA@RIKEN & 2010 & 8 & $4.5-15 / 1-3$ & 60 & 1 \\
\hline Fermi@ELETTRA & 2010 & 2.4 & $0.01-0.06 / 200-1000$ & 10 & 1 \\
\hline SwissFEL & 2016 & 5.8 & $12 / 1$ & 100 & $2 @ 50 \mathrm{~ns}$ \\
\hline Eu-XFEL & $>2017$ & 17.5 & $0.4-20 / 0.5-30$ & 10 & $2700 @ 220 \mathrm{~ns}$ \\
\hline LCLSII & $>2020$ & $4-14.5$ & $0.2-25 / 0.5-60$ & $120-10^{6}$ & 1 \\
\hline
\end{tabular}

Table 1: main features of some existing and future FEL facilities.

Web $[8,9,10]$. In general, FEL facilities have several beam lines differing in photon energy range and addressing different types of experiments. FEL operation may change significantly from one facility to the other. For example, while the European XFEL (also known as Eu-XFEL) will produce trains of high repetition rate $(4.5 \mathrm{MHz}) \mathrm{X}$-rays, with a $100 \mathrm{~ms}$ interval between two subsequent trains, the LCLS in Stanford is operated at a constant pulse rate of $120 \mathrm{~Hz}$. Table 1 shows the main features of a few FELs, some already operational, some other still under construction. All of the listed FEL characteristics and operation modes have an impact on the instrumentation design choices. Actually, in the present stage of microelectronic technology, it is very hard, if possible at all, to conceive a device capable either of covering the entire range of photon energies at an individual FEL, or of adapting to the operating mode of FELs at different facilities. A specific design has generally to be adopted for each particular beam line at each particular FEL.

\section{Instrumentation for X-ray FELs}

Although each experiment at FELs requires a specific detection system, two main scientific cases may be identified:

- energy sensitive detectors with Fano limited energy resolution for spectroscopic experiments (0D), possibly featuring position sensitivity for angular (wavelength) dispersive experiments (1D); these include

- silicon drift detectors,

- high-Z detectors,

- cryogenic detectors;

- area detectors for imaging experiments, based on X-ray diffraction techniques (2D); these include

- charge coupled devices (CCD),

- hybrid pixel detectors, 
- CMOS monolithic active pixel sensors (MAPS).

The present review will concentrate on the second family of detectors.

\section{Bi-dimensional X-ray imagers for present and next generation FELs}

Instruments for experiments at FEL facilities are, at present, in different stages of development, depending, among other factors, on the expected start-of-operation date of the machine they are being built for. X-ray imagers features are adjusted to the specific characteristics of the FEL beam line they will be working on. In particular, the X-ray pulse rate has a strong impact on the choice of the detector technology. Some FELs already in operation, like the LCLS in Stanford, USA, or the SACLA, in Japan, feature pulse rates not exceeding $120 \mathrm{~Hz}$. In this case, even considering the typically large size of the detector, 1 Mpixel or larger, the entire image resulting from one X-ray pulse can be acquired before the next one arrives. The solutions proposed for this type of facilities are mostly based on CMOS MAPS (including silicon on insulator, SOI) or CCD technologies, which, while being characterized by a relatively small processing speed, are commonly used in imaging applications and can easily accommodate pixel pitches below $50 \mu \mathrm{m}$. A different approach is required in the case of the extremely fast rates foreseen for some of the FELs currently under construction, like the European XFEL with its particular beam structure (already mentioned in Section 2). In this environment, hybrid pixel technologies, successfully employed in high energy physics applications, can guarantee the speed needed to process data in the time between two subsequent pulses ( $220 \mathrm{~ns}$ ). On the other hand, the bandwidth required for direct data readout soon after processing (in the case of a 10 bit A-to-D in pixel conversion, 1 Mpixel $\times 10$ bits/220 ns $\simeq 50 \mathrm{~Tb} / \mathrm{s}$ ) is not within the reach of any known technology. A store-locally/read-out-later operation mode is therefore adopted by the three groups working in the European XFEL consortium, whose activity and results are discussed below in Section 4.2. This operation mode consists of storing as many data as possible for the pulse train duration and sending them to the acquisition system during the inter-train interval. In the following paragraph, an overview of the state of the art in the $2 \mathrm{D}$ X-ray instrumentation area is given, limited to the most significant examples taken from the large set of solutions proposed for experiments at FELs. Detectors will be arbitrarily classified according to the pulse rate of the FELs they have been or are being designed for.

\subsection{Detectors for slow pulse rate FELs}

This section provides a few representative cases of detectors developed (or under development) for imaging applications at LCLS and SACLA. As mentioned above, they are based on CMOS MAPS or CCD technologies.

\subsubsection{The Percival project}

The Pixellated Energy Resolving CMOS Imager, Versatile and Large (Percival) [11] is a monolithic pixel sensor. The design is based on a quadruple well $180 \mathrm{~nm}$ CMOS process, providing a $12 \mu \mathrm{m}$ thick high resistivity epitaxial layer, which represents the sensitive volume of the detector. This sets the maximum detectable photon energy to a few $\mathrm{keV}$. On the other side of the energy range, the small equivalent noise charge (about 15 electrons) makes it possible to detect photons 
with energy as small as a few hundreds of eV. The operation of the readout channel is based on adaptive gain switching between the collecting diode and three capacitors of increasing size. This gives single photon discrimination at low flux, while retaining the capability to measure a higher flux when required at the cost of increased noise. The project is pursuing the ambitious goal to fabricate a $25 \mu \mathrm{m}$ pitch, $4 \mathrm{k} \times 4 \mathrm{k}$ pixel detector to be read out at a frame rate of $120 \mathrm{~Hz}$. This would make the device suitable for operation at the LCLS.

\subsubsection{The Xamps detector}

Xamps is a monolithic pixel detector (with a $90 \mu \mathrm{m}$ pitch) fabricated in a high resistivity substrate and read out by a multichannel ASIC [12]. JFET switches integrated in the sensing layer are used to operate the system according to an accumulation and a data readout mode. During the first phase, the switches are open and the charge released by a photon in the substrate of the sensor (400 $\mu \mathrm{m}$ thick) is collected by a capacitor taking up most of the pixel area. A few microseconds are needed to readout the $3.5 \mathrm{pC}$ charge corresponding to the full well capacitance. During the second phase, the switches are closed, therefore allowing the charge to flow to the readout lines. In order to cope with the wide input dynamic range, amplitude measurements are performed in two stages. A first, coarse digitization is performed on-chip. Residuals from the conversion are digitized with an external 14-bit ADC. A few milliseconds are needed to readout the entire detector, making it suitable for applications at FELs continuously operated at rates in the $100 \mathrm{~Hz}$ range, like the LCLS.

\subsubsection{The SOPHIAS detector}

The Silicon-On-Insulator PHoton Imaging Array Sensor (SOPHIAS) is an X-ray pixel detector based on a CMOS silicon-on-insulator technology targeting X-ray diffraction imaging experiments [13]. The detector, featuring a $30 \mu \mathrm{m}$ pitch, is designed to be sensitive to single photons in the 5.5-7 keV energy interval and to perform frame readout at a $60 \mathrm{~Hz}$ rate, suitable for operation at the Sacla FEL. Charge is processed using a typical CMOS MAPS scheme. The relatively low degree of radiation hardness typical of this technology is strongly mitigated by the shielding action of the $500 \mu \mathrm{m}$ thick handle wafer also acting as the sensitive volume of the detector. A dual-gain concept is used in the SOPHIAS detector to address the issue of wide input dynamic range, similarly to the case of the Percival detector. The basic chip includes as many as about 1.9 Mpixels.

\subsubsection{The HIPPO readout chip for CCD detectors}

In general, CCDs represent a natural choice for imaging applications. However, in FEL applications, suitable architectures have to be envisioned to overcome the limited readout rates (of the order of a few frames per second) typical of sequentially read out structures. For this purpose, almost-fully- or fully-column-parallel architectures have been proposed for applications at the LCLS FEL experiments [14]. Front-end chips may be needed to provide amplification, sampling and fast digitization of the charge collected by the detector. The High-speed Image Preprocessor with Oversampling (HIPPO) is a column-parallel CCD readout ASIC designed in a $65 \mathrm{~nm}$ CMOS technology [15]. The implemented ASIC consists of 16 channels subdivided in four modules of four channels each. Each module includes 4 analog front-end blocks and four sample-and-hold circuits. A-to-D conversion is performed through a pipeline ADC shared among four channels. Data from the ADC are sent off chip at a $480 \mathrm{Mb} / \mathrm{s}$ rate. 


\subsection{Detectors for fast pulse rate FELs}

This section presents some examples of detectors being developed for application to experiments at FELs with fast X-ray pulse rate. One FEL with this property is the European XFEL, already mentioned many times above. In particular, the Eu-XFEL consortium is funding three independent detector development projects. Each of the three projects is proposing a different solution to the challenges posed by the characteristics of the Eu-XFEL beams, in particular the wide signal dynamic range (from 1 to 10000 photons @ $1 \mathrm{keV}$ ) and the high frame rate [16]. In addition, the Italian PixFEL project, at a much earlier stage than the ones funded by the Eu-XFEL Consortium, is also developing a fast, small pitch pixel detector for diffraction imaging.

\subsubsection{The AGIPD detector}

The AGIPD (Adaptive Gain Integrating Pixel Detector) project [17] consists of a hybrid pixel array, with a readout ASIC (designed in a $130 \mathrm{~nm}$ CMOS process) bump-bonded to a silicon sensor. The front-end channel takes advantage of a dynamic gain switching technique to cover the large dynamic range of the input signal, while an in-pixel analog pipeline is used to store the images recorded during the $0.6 \mathrm{~ms}$ long pulse train. About 200 images per train can be recorded by the detector, corresponding to the about 200 storage capacitors integrated in the pixel area, which is $200 \mu \mathrm{m} \times 200 \mu \mathrm{m}$. One of the major concerns in this approach is the design of the switches, which should be such to enable charging of the capacitors in the $200 \mathrm{~ns}$ inter-bunch period while preventing them from discharging through leakage during the $100 \mathrm{~ms}$ inter-train period. The main scientific applications are coherent X-ray imaging and photon correlation spectroscopy at $12 \mathrm{keV}$ photon energies.

\subsubsection{The LPD detector}

The approach of the LPD (Large Pixel Detector) project [16] to the issue of large input dynamic range involves employing three different channels in parallel, each with a different gain setting and followed by its own analog pipeline. In order for the pixel to incorporate three separate pipelines with 500 storage capacitors each, the cell pitch needs to be relatively large, about $500 \mu \mathrm{m}$ (which may be too large for some applications). The LPD front-end module will also include an interposer in order to adapt the pitch difference between the silicon sensor and the ASIC. This solution makes it possible to use a different pitch on the two interconnected layers and offers the advantage of adding an extra radiation shield, therefore relaxing the radiation hardness requirements on the ASIC. However, it may contribute some parasitic capacitance at the channel input, resulting in a degradation of the noise performance. The three analog pipelines can also be linked together to form a single, 1500-deep pipeline, amounting to about half of the number of images that can be taken in a single pulse train at the European XFEL. The LPD focuses on the $12 \mathrm{keV}$ photon energy range, optimally suited for the so-called liquid scattering experiments.

\subsubsection{The DSSC detector}

The DEPFET Sensor with Signal Compression (DSSC) [18] uses a nonlinear response in the sensing element of the pixel to cope with the large dynamic range, and a digital memory to store images inside the pixel. While being one of the original points of the proposal, implementing the 
non linear response in the DEPFET sensor requires quite a complex fabrication process, resulting in fairly long turnaround times. On the other hand, the very low noise performance of the DEPFET makes it an excellent candidate for experiments using low energy $\mathrm{X}$-rays, around $1 \mathrm{keV}$ or smaller. The DSSC detector is made of hexagonal pixels with a $136 \mu \mathrm{m}$ side, yielding a bump bond pitch of about $200 \mu \mathrm{m}$. Use of hexagonal pixels offers the threefold advantage of improving the homogeneity of the field in the substrate, minimizing the collection time and reducing the charge sharing. The front-end chip is designed in a $130 \mathrm{~nm}$ CMOS process provided by IBM and will include $64 \times 64$ elements. Eight ASICs will be bump-bonded to a monolithic DEPFET sensor. Compared to the two solutions above, in the DSSC detector data are directly digitized on-chip with a high speed, 8-bit, single-ramp (Wilkinson) A-to-D converter, whose operation is based on a $800 \mathrm{MHz}$ clock (the content of the ADC counter is actually incremented on both fronts of the clock signal). The digital memory consists of about 600 cells per pixel.

\subsubsection{The PixFEL project}

The PixFEL project, funded by INFN for the 2014-2016 period, aims to develop the main microelectronic building blocks and investigating the enabling technologies for X-ray diffraction imagers at FELs [19]. The long term goal of the project is the fabrication of an advanced 2D X-ray camera for CXDI measurements, targeting the stringent requirements of experiments at future Xray FEL facilities, like the Eu-XFEL. The detector is supposed to consist of a multilayer structure resulting from the vertical interconnection of the sensor to a dual-tier front-end chip. Use of a dual-tier approach for the front-end chip has the main purpose of increasing the functional density. In particular, one layer is used to accommodate the analog front-end channel and the ADC, while memory cells are integrated in the second layer together with the digital readout electronics. The collaboration has already designed and tested a prototype front-end channel, fabricated in a $65 \mathrm{~nm}$ CMOS technology and tailored for photon energies between $1 \mathrm{keV}$ and $10 \mathrm{keV}$. The front-end channel includes a charge preamplifier with $80 \mathrm{~dB}$ input dynamic range, a time variant shaper and a 10 bit ADC in a $110 \mu \mathrm{m}$ pitch. The operation of the charge preamplifier is based on the non-linear behavior of a MOS capacitor in the feedback network [20]. The detector is based on an active edge pixel sensor [21, 22], with a substrate thickness of $450 \mu \mathrm{m}$ for a $90 \%$ detection efficiency at photon energies of $10 \mathrm{keV}$. Assuming a sensor made up of ladders $2.56 \mathrm{~cm} \times 5.12 \mathrm{~cm}$ in area and a dead region of $200 \mu \mathrm{m}$ on each of the four sides (including the guard ring structure and the mechanical tolerance in the detector assembly), an overall dead area as small as $2.3 \%$ can be achieved.

\section{Conclusion}

$\mathrm{X}$-rays have been a very powerful probing tool in many fields since their discovery. The advent of free electron lasers opens up new possibilities to probe matter using X-rays with unprecedented features in terms of beam brightness, pulse speed and energy tuneability. New instrumentation is needed to comply with the challenging requirements of the experiments at FELs. The development of innovative detectors can take advantage of the continuous advancement of microelectronic technologies (scaled CMOS and vertical integration processes, active edge detectors) and of already tested solutions that can be borrowed from the high energy physics community. The interest in the field of instrumentation for FELs has been steadily growing in the past few years, together with the 
number of new facilities around the world, and is very likely to continue growing in the years to come.

\section{Acknowledgements}

The author would like to thank his colleagues in the PixFEL collaboration for helpful discussions and ideas, of crucial importance to get a deeper understanding of the main issues in instrumentation for photon science applications.

\section{References}

[1] M. M. Seibert, Nature 47078 (2011).

[2] J. Miao, Annu. Rev. Biophys. Biomol. Struct. 33157 (2004).

[3] H.N. Chapman, Proceedings of FEL 2006, Bessy, Berlin, Germany, Aug. 27 - Sep. 1 2006, 805.

[4] Q. Shen, Journal of Synchrotron Radiation 11432 (2004).

[5] S.M. Gruner, Rev. Sci. Instrum. 731402 (2002).

[6] B.E. Carlsten, J. Phys. B: At. Mol. Opt. Phys. 47234012 (7pp) (2014).

[7] V. Malka, Nature Physics 4447 (2008).

[8] LCLS-II New Instruments Workshops Report [Online]. Available: http://slac.stanford.edu/pubs/slacreports/reports19/slac-r-993.pdf.

[9] Ultrafast Phenomena at the Nanoscale: Science opportunities at the SwissFEL X-ray Laser [Online]. Available: http://www.psi.ch/swissfel/CurrentSwissFELPublicationsEN/

/SwissFEL_Science_Case_small.pdf.

[10] Research possibilities at the European XFEL [Online]. Available: https://www.xfel.eu/research/possibilities.

[11] C.B. Wunderer, JINST 9 C03056 (2014).

[12] G.A. Carini, IEEE 2009 Nuclear Science Symposium Conference Record 2151 (2009).

[13] T. Hatsui, 2013 International Image Sensor Workshop (IISW), Snowbird, Utah, USA, June 12-16, 2013.

[14] D. Doering, Rev. Sci. Instrum., 82 (7) 073303 (8pp) (2011).

[15] C.R. Grace, IEEE 2011 Nuclear Science Symposium Conference Record 1435 (2011).

[16] H. Graafsma, JINST 4 P12011 (2009).

[17] B. Henrich, Nucl. Instrum. Methods A 633511 (2011).

[18] M. Porro, Nucl. Instrum. Methods A, 624509 (2010).

[19] G. Rizzo, JINST 10 C02024 (2015).

[20] M. Manghisoni, IEEE Trans. Nucl. Sci. 62 (5) 2318 (2015).

[21] J.C. Kenney, IEEE Trans. Nucl. Sci. 48 (6) 2405 (2001).

[22] G.-F. Dalla Betta, 2011 IEEE Nuclear Science Symposium Conference Record 1334 (2011). 